Research Article

\title{
An Evolutionary Game Analysis of Internet Public Opinion Events at Universities: A Case from China
}

\author{
Hongying Wen $\left(\mathbb{D},{ }^{1}\right.$ Kairong Liang $\mathbb{D}^{2},{ }^{2}$ and Yiquan $\mathrm{Li} \mathbb{D}^{1,2}$ \\ ${ }^{1}$ College of Marxism, Fuzhou University, Fuzhou, Fujian 350108, China \\ ${ }^{2}$ School of Economics and Management, Fuzhou University, Fuzhou, Fujian 350108, China \\ Correspondence should be addressed to Yiquan Li; xgb@fzu.edu.cn
}

Received 14 March 2020; Revised 5 June 2020; Accepted 12 June 2020; Published 17 July 2020

Academic Editor: Dimitris Mourtzis

Copyright (c) 2020 Hongying Wen et al. This is an open access article distributed under the Creative Commons Attribution License, which permits unrestricted use, distribution, and reproduction in any medium, provided the original work is properly cited.

\begin{abstract}
Internet public opinion events at universities in China occurred frequently, creating painful repercussions for reputation and stability of colleges and universities. To better cope with the problem, this paper explores an evolutionary mechanism of the university Internet public opinion events. Firstly, we discuss the interactions and behavior of three key participants: an Internet medium, university students as a whole, and administration. Secondly, we construct a tripartite evolutionary game model consisting of an Internet medium, student group, and university administration and then analyze and obtain the differential dynamic equations and equilibrium points. Subsequently, the evolutionary stable equilibrium is further analyzed. Finally, we employ numerical studies to examine how the tripartite behavior choices affect evolutionary paths and evolutionary equilibrium strategies. Results are derived as follows: under certain conditions, there exists an asymptotically stable equilibrium point for the tripartite evolutionary game. On the one hand, appropriate penalties and rewards should be provided to foster objectives and fair behaviors of the network medium. On the other hand, university students should be educated and guided to deal rationally with negative effects of Internet public opinion events. Moreover, online real-name authentication is an important and necessary measure. Finally, the university administration should release truthful, timely, and comprehensive information of Internet public opinion events to mitigate potential negative impacts.
\end{abstract}

\section{Introduction}

In recent years, Internet public opinion events occurred frequently across colleges and universities in China. For example, several incidents recently made to headlines on the Internet social media such as "South China University of Technology tampered postgraduate entrance exam scores" [1] and "Foreign students' study partners at the Shandong University" [2]. The resulting negative public opinions arose from the students on the campus and spread rapidly throughout China, bringing reputation and public relation damage to the universities involved in these events.

With rapid development of new generations of information technology, new social media on the Internet, such as WeChat, Weibo, and mobile apps, have become popular among college and university students in China. Due to real- time spread, interactive nature, and pervasiveness of the new social media, university students can gain easy access to and add their personal comments on public opinion events, further aggravating their abrupt development and amplifying their negative impacts. A series of great challenges have been created for university administrations to properly guide and handle these events. Furthermore, facing miscellaneous and sometimes false Internet information about the events, Chinese university administrations typically suffer from lack of public opinion response tools and proper handling methods. Hence, mishandling of and inappropriate response to Internet public opinion events by Chinese university administrations can quickly turn to polarized group protests or mass incidents.

In order to better deal with Internet public opinion events at colleges and universities in China, university 
administrations should enhance their understanding of multiple drivers behind online public opinion events and study their evolution and communication mechanisms. This paper aims to address this challenge by developing a tripartite evolutionary game model consisting of a network medium, university student group, and university administration. This model allows us to analyze the impacts of the three participants' behavior on evolutionary paths and equilibrium points.

The rest of this paper is organized as follows. Section 2 briefly reviews literature related to this research. In Section 3, we first propose a tripartite evolutionary game to model public opinion events surrounding an Internet medium, university student group, and university administration. Then, we derive the replicator dynamic system and evolutionary stable equilibrium and discuss stability of the equilibrium points. A numerical example is given in Section 4 to analyze the spread mechanism of Internet public opinion events at universities in China. Finally, Section 5 concludes the paper with future research directions.

\section{Literature Review}

Two streams of literature are closely related to our research: Internet public opinion events and evolutionary game models for these events.

2.1. Network Public Opinion. With rapid development of Internet, online public opinion has become one of the hotspots in information management and has attracted more and more attention of many scholars. For instance, Laxmidhar et al. [3] developed a Sznajd model with threedimensional networks and scale-free networks. Weisbuch [4] investigated how different social network topologies affect the dynamics of the bounded confidence model. Fortunato [5] studied a damage spreading problem characterized by the Krause-Hegselmann consensus model with a scale-free Barabasi-Albert network. Mare et al. [6] applied strategic game theory to simulate the interpersonal persuasion process between two individuals. Cao and $\mathrm{Li}$ [7] analyzed the individual persuasion process by using a gender game. Their research revealed that the system will either reach a final consensus with the initial dominant view or a steady state in which multiple viewpoints coexist. Liu et al. [8] studied supervision and evolution mechanisms of public opinion information and analyzed the impacts of the punishment coefficient, risk coefficient, network node strength, and neighbor node selection preference on individual selection of rumor propagation behavior. Li et al. [9] developed an evolutionary game model to examine the rumor propagation process in a complex network. Jiang et al. [10] provided a postevaluation method to convert Internet nonstructural public in Internet crisis events. Ai et al. [11] first analyzed geographical interaction characteristics of online public opinions on WeChat and then proposed different management methods for coping with different online public opinions based on the economic, historical, and political characteristics in different regions. Wang et al. [12] applied a bimodal Gaussian model to examine the key characteristics of Internet public opinion information transmission and revealed the spread mechanism of social events on the Internet.

The aforesaid literature review reveals that the current research on online public opinions has been widely studied. However, existing research rarely considers participants' bounded rationality. In reality, participants in a university online public opinion event such as network media, university students, and university administration are typically not completely rational and often respond to the event irrationally. This is particularly true for university students who are easily agitated and follow the herd without a second thought. In contrast, we put forward a tripartite evolutional game model with bounded rationality to characterize the evolution and communication processes of Internet public opinions. We also offer measures to manage bounded rationality in such events.

\subsection{Evolutionary Game Analysis of Internet Public Opinion.} This paper resorts an evolutionary game method to analyze Internet public opinion events with three participants. An evolutionary game is an effective approach to analyze the behavioral paradigm of bounded rationality under incomplete information. In an evolutionary game, players often cannot obtain full information for all participants. They constantly adjust their behavior strategy to improve their payoffs based on the principle of profit maximization. The core concepts in an evolutionary game are "evolutionary stable strategy" and "replication dynamics" [13].

Due to the superiority of evolutionary games in behavior analysis, they have been applied to study the evolution and control of Internet public opinions. For instance, Li et al. [14] and Xiao et al. [15] studied the influencing factors and diffusion mechanisms of Internet public opinions by evolutionary games. Based on the attitude change theory, group behavior theory, and evolutionary game theory, Yin et al. [16] proposed an agentbased online opinion formation model in the perspective of sociology and psychology. Askarizadeh et al. [17] developed an evolutionary game model to analyze the spread and control of rumors in social networks. These existing studies typically consider two players (i.e., network media and regulator) in the game. But, it is difficult to accurately describe the increasingly complex behavior patterns of the participants in Internet public opinions. In a typical university Internet public opinion event in China, participants usually consist of Internet media, university students, and administrations. To better characterize the complex behavior characteristics of the rapid spread, heterogeneous participants, and irrational response in these events, this paper develops a tripartite evolutionary game model consisting of an Internet medium, university students, and administration. This model allows us to reveal the spread mechanism of university Internet public opinion events in China, thereby formulating proper measures to mitigate potential damage. 


\section{Modeling}

In this section, we first establish an evolutionary game model consisting of three players, an Internet medium, university student group, and university administration for a university online public opinion event. Then, we analyze the interactions among the players and their equilibrium strategies.

3.1. Problem Descriptions and Assumptions. In a university Internet public opinion event, we consider three bounded rational participants, an Internet medium, university students as a group, and university administration. Upon naively selecting its strategy, a participant rationally makes decision to maximize its payoff function associated with the chosen strategy. That is, all participants optimize their strategies based on their previous decision and other participants' interactive response [18]. For model tractability, we make the following assumptions.

Assumption 1. The network medium has two strategies of "Reporting" and "No-reporting" $\left\{m_{1}, m_{2}\right\}$ with $(p, 1-p)$ as the corresponding probabilities. Which strategy that the Internet medium chooses depends on the medium flow revenue from the Internet public opinion events. If the Internet medium takes the $m_{1}$ strategy to report the event, it gets a fixed revenue $\alpha_{12}$ and pays a fixed cost $\beta_{10}$. If the medium does not correctly report the event (i.e., reporting false information or information out of the context), it will incur a social welfare loss $\beta_{12}$. In addition, if university students and university administration take part in the Internet public opinion event, then the Internet medium will receive extra revenues $\alpha_{21}$ and $\alpha_{31}$, respectively, with heated debates of the public opinion event. Otherwise, the network medium loses $\beta_{11}$ if it chooses not to report the event.

Assumption 2. For university students as a group player, it can choose two strategies of "participation" and "nonparticipation" $\left\{s_{1}, s_{2}\right\}$ with $(x, 1-x)$ as the corresponding probabilities. University students can gain a social identity revenue $\alpha_{21}$ with a searching and time cost $\beta_{20}$. Moreover, university students obtain an extra revenue $\alpha_{22}$ if their participation in the event meets with a positive appraisal from the university administration. On the contrary, if their participation meets with disciplines by the university administration, they will incur an individual misjudgment cost $\beta_{21}$, university penalty loss $\beta_{22}$, and social cost $\beta_{23}$ owing to spreading rumors and misinformation.

Assumption 3. For the university administration, its strategy space is (positive response, negative response), denoted by $\left\{u_{1}, u_{2}\right\}$, with $(y, 1-y)$ as the corresponding probabilities. When the university administration adopts the positive strategy, its revenue is $\alpha_{30}$ due to enhanced reputation subject to an operation cost $\beta_{30}$. The university administration can obtain an additional revenue $\alpha_{31}$ by actively participating in the online interactions surrounding the event. In addition, if university students objectively spread information, the university administration can obtain an extra revenue $\alpha_{32}$. On the other hand, if the university administration takes the negative strategy, it incurs a loss $\beta_{31}$.

To summarize, the notation used in this paper is depicted in Table 1.

$$
\begin{aligned}
& M=\left(\begin{array}{llll}
M_{11} & M_{12} & M_{13} & M_{14} \\
M_{21} & M_{22} & M_{23} & M_{24}
\end{array}\right) \\
& =\left(\begin{array}{cccc}
\alpha_{10}+\alpha_{11}+\alpha_{12}-\beta_{10}-\beta_{12} & \alpha_{10}+\alpha_{12}-\beta_{10}-\beta_{12} & \alpha_{11}+\alpha_{12}-\beta_{10}-\beta_{12} & \alpha_{12}-\beta_{10}-\beta_{12} \\
-\beta_{11} & -\beta_{11} & -\beta_{11} & -\beta_{11}
\end{array}\right) \text {, } \\
& S=\left(\begin{array}{llll}
S_{11} & S_{12} & S_{13} & S_{14} \\
S_{21} & S_{22} & S_{23} & S_{24}
\end{array}\right)
\end{aligned}
$$

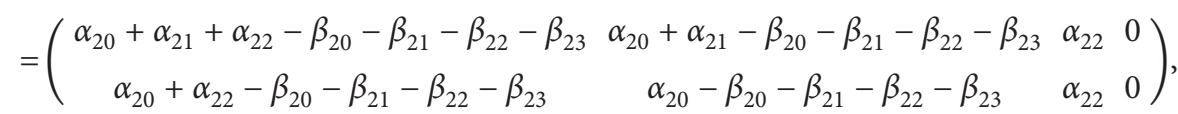

$$
\begin{aligned}
& U=\left(\begin{array}{llll}
U_{11} & U_{12} & U_{13} & U_{14} \\
U_{21} & U_{22} & U_{23} & U_{24}
\end{array}\right) \\
& =\left(\begin{array}{cccc}
\alpha_{30}+\alpha_{31}+\alpha_{32}-\beta_{30} & -\beta_{31} & \alpha_{30}+\alpha_{31}-\beta_{30} & -\beta_{31} \\
\alpha_{30}+\alpha_{32}-\beta_{30} & -\beta_{31} & \alpha_{30}-\beta_{30} & -\beta_{31}
\end{array}\right) .
\end{aligned}
$$

3.2. Model Formulation. Firstly, this section discusses the payoff table of the tripartite game. If the Internet media $(m)$, university students $(s)$, and university administration $(u)$ all choose their first strategy, the resulting situation is represented by $\left(m_{1}, s_{1}, u_{1}\right)$. The corresponding payoffs are denoted by $M_{11}, S_{11}$, and $U_{11}$, respectively. Similarly, the payoffs under the other seven situations can be expressed as shown in Table 2.

Given each player's two strategies, Table 2 shows the 8 possible situations under different combinations of the player's strategic choices. Based on the assumptions in Section 3.1, the payoff matrices of the network medium $(m)$, university 
TABLE 1: Notations and their meaning.

\begin{tabular}{lcc}
\hline Participants & Notation & Meaning \\
& $\alpha_{10}$ & Reporting probability \\
Network media & $\alpha_{11}$ & Additional benefits from university student participation \\
& $\alpha_{12}$ & Additional benefits from university administration participation \\
& $\beta_{10}$ & Gains from advertising, clicks, and user loyalty \\
Information disclosure, follow-up costs
\end{tabular}

Note. $\alpha$ and $\beta$ denote, respectively, the revenues and costs.

TABle 2: Tripartite game normative representation.

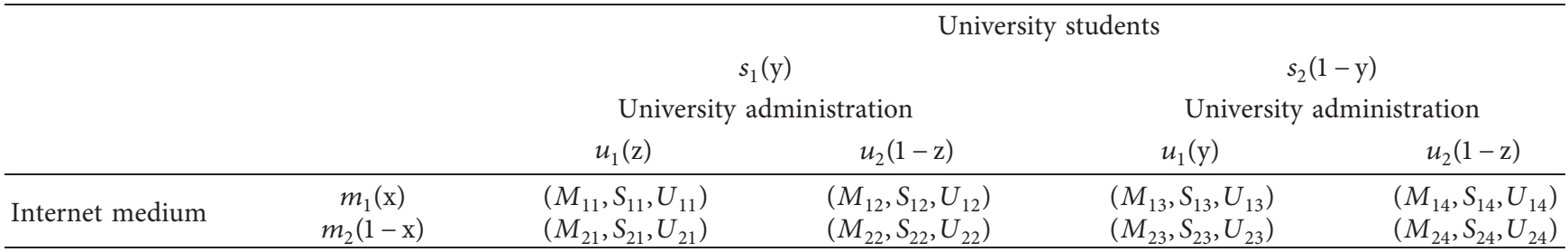

students $(s)$, and university administration $(u)$ are denoted by $M, S$, and $U$, respectively, and determined as follows:

Next, given the probability distributions, the expected payoffs of the three participants are analyzed.

Section 3.1 assumes that the probability of university students participating in the event is $y$, the probability of the university administration responding positively is $z$, and the probability of the Internet medium reporting the event is $p$.

For the Internet medium, its expected payoff under strategy $m_{1}$ is

$$
\begin{aligned}
E\left(m_{1}\right)= & y z M_{11}+y(1-z) M_{12}+(1-y) z M_{13} \\
& +(1-y)(1-x) M_{14} .
\end{aligned}
$$

Its expected payoff under strategy $m_{2}$ is

$$
\begin{aligned}
E\left(m_{2}\right)= & y z M_{21}+y(1-z) M_{22}+(1-y) z M_{23} \\
& +(1-y)(1-z) M_{24} .
\end{aligned}
$$

As such, the Internet medium's overall expected payoff is

$$
E(x)=x E\left(m_{1}\right)+(1-x) E\left(m_{2}\right) \text {. }
$$

Similarly, the expected payoffs of university students and university administration are shown in equations (5)-(7) and equations (8)-(10), respectively.
For university students, its expected payoffs under strategies $s_{1}$ and $s_{2}$ are, respectively,

$$
E\left(s_{1}\right)=x z S_{11}+x(1-z) S_{12}+(1-x) z S_{21}+(1-x)(1-z) S_{22} \text {, }
$$

$E\left(s_{2}\right)=x z S_{13}+x(1-z) S_{14}+(1-x) z S_{23}+(1-x)(1-z) S_{24}$.

The university students' overall expected payoff is thus

$$
E(y)=y E\left(s_{1}\right)+(1-y) E\left(s_{2}\right) \text {. }
$$

For the university administration, its expected payoffs under strategies $u_{1}$ and $u_{2}$ are, respectively,

$$
\begin{aligned}
E\left(u_{1}\right)= & x y U_{11}+x(1-y) U_{13}+(1-x) y U_{21} \\
& +(1-x)(1-y) U_{23}, \\
E\left(u_{2}\right)= & x y U_{12}+x(1-y) U_{14}+(1-x) y U_{22} \\
& +(1-x)(1-y) U_{24} .
\end{aligned}
$$

The university administration's expected payoff is

$$
E(z)=z E\left(u_{1}\right)+(1-z) E\left(u_{2}\right)
$$


3.3. Solution of the Evolutionary Game. In order to analyze the long-term behavior and equilibrium strategies of the three participants, their replicator dynamics equations are shown below based on equations (2)-(10):

$$
\left\{\begin{array}{l}
\frac{\mathrm{d} x}{\mathrm{~d} t}=M(x)=x\left(E\left(m_{1}\right)-E(x)\right)=x(1-x)\left(y \alpha_{10}+z \alpha_{11}+\alpha_{12}-\beta_{10}+\beta_{11}-\beta_{12}\right), \\
\frac{\mathrm{d} y}{\mathrm{~d} t}=S(y)=y\left(E\left(s_{1}\right)-E(y)\right)=y(1-y)\left[\alpha_{20}+x \alpha_{21}+z \alpha_{22}-\beta_{20}-\beta_{21}-\beta_{22}-\beta_{23}\right], \\
\frac{\mathrm{d} y}{\mathrm{~d} t}=U(z)=z\left(E\left(u_{1}\right)-E(z)\right)=z(1-z)\left(\alpha_{30}+x \alpha_{31}+y \alpha_{32}+\beta_{31}-\beta_{30}\right) .
\end{array}\right.
$$

According to the equilibrium theory, we have

$$
\begin{aligned}
& \frac{\mathrm{d} x}{\mathrm{~d} t}=0, \\
& \frac{\mathrm{d} y}{\mathrm{~d} t}=0, \\
& \frac{\mathrm{d} z}{\mathrm{~d} t}=0 .
\end{aligned}
$$

By solving equation (12), it is easy to obtain 8 pure strategy equilibrium points and the mixed strategy equilibrium of the tripartite evolutionary game as $E_{1}(0,0,0)$, $E_{2}(0,0,1), E_{3}(0,1,0), E_{4}(0,1,1), E_{5}(1,0,0), E_{6}(1,0,1)$, $E_{7}(1,1,0), E_{8}(1,1,1)$, and $E_{9}=\left(x^{*}, y^{*}, z^{*}\right)$. Let $a_{1}=a_{10}$, $a_{2}=a_{11}, \quad a_{3}=\alpha_{12}-\beta_{10}+\beta_{11}-\beta_{12}, \quad b_{1}=\alpha_{20}, \quad b_{2}=\alpha_{21}$, $b_{3}=\alpha_{20}-\beta_{20}-\beta_{21}-\beta_{22}-\beta_{23}, \quad c_{1}=\alpha_{31}, \quad c_{2}=\alpha_{32}, \quad$ and $c_{3}=\alpha_{30}+\beta_{31}-\beta_{30}$. We obtain the mixed strategy equilibrium point as

$$
E_{9}\left(x^{*}, y^{*}, z^{*}\right)=\left(\frac{-a_{1} b_{2} c_{3}-a_{2} b_{3} c_{2}+a_{3} b_{2} c_{2}}{a_{1} b_{2} c_{1}+a_{2} b_{1} c_{2}}, \frac{a_{2} b_{3} c_{1}-a_{3} b_{2} c_{1}+a_{2} b_{1} c_{3}}{a_{1} b_{2} c_{1}+a_{2} b_{1} c_{2}}, \frac{a_{1} b_{1} c_{3}-a_{1} b_{3} c_{1}-a_{3} b_{1} c_{2}}{a_{1} b_{2} c_{1}+a_{2} b_{1} c_{2}}\right) .
$$

By solving equation (14),

$$
\left\{\begin{array}{l}
y \alpha_{10}+z \alpha_{11}+\alpha_{12}-\beta_{10}+\beta_{11}-\beta_{12}=0, \\
\alpha_{20}+x \alpha_{21}+z \alpha_{22}-\beta_{20}-\beta_{21}-\beta_{22}-\beta_{23}=0 \\
\alpha_{30}+x \alpha_{31}+y \alpha_{32}+\beta_{31}-\beta_{30}=0 .
\end{array}\right.
$$

In $E_{9}\left(x^{*}, y^{*}, z^{*}\right)$, if none of the players selects a mixed strategy, i.e., $x^{*} \notin(0,1), y^{*} \notin(0,1)$, and $z^{*} \notin(0,1)$, then there does not exist a mixed strategy equilibrium point. If any one of the participants chooses pure strategy 0 or 1 in the mixed strategy (note that if any two participants select pure strategies, then equation (14) has no solution. In this case, there will be no mixed strategy equilibrium point), then there exist 6 special mixed strategy equilibrium points $\left(x^{*}, y^{*}, z^{*}\right)$ as follows:

$$
\begin{aligned}
& \left(0, \frac{\beta_{30}-\beta_{31}-\alpha_{30}}{\alpha_{32}}, \frac{\beta_{20}+\beta_{21}+\beta_{22}+\beta_{23}-\alpha_{20}}{\alpha_{22}}\right), \\
& \left(1, \frac{\beta_{30}-\beta_{31}-\alpha_{30}-\alpha_{31}}{\alpha_{32}}, \frac{\beta_{20}+\beta_{21}+\beta_{22}+\beta_{23}-\alpha_{20}-\alpha_{21}}{\alpha_{22}}\right), \\
& \left(\frac{\beta_{30}-\beta_{31}-\alpha_{30}}{\alpha_{31}}, 0, \frac{\beta_{10}+\beta_{12}-\beta_{11}-\alpha_{12}}{\alpha_{11}}\right), \\
& \left(\frac{\beta_{30}-\beta_{31}-\alpha_{30}-\alpha_{32}}{\alpha_{31}}, 1, \frac{\beta_{10}+\beta_{12}-\beta_{11}-\alpha_{12}-\alpha_{10}}{\alpha_{11}}\right), \\
& \left(\frac{\beta_{20}+\beta_{21}+\beta_{22}+\beta_{23}-\alpha_{20}}{\alpha_{21}}, \frac{\beta_{12}+\beta_{10}-\beta_{11}-\alpha_{12}}{\alpha_{10}}, 0\right), \\
& \left(\frac{\beta_{20}+\beta_{21}+\beta_{22}+\beta_{23}-\alpha_{20}-\alpha_{22}}{\alpha_{21}}, \frac{\beta_{12}+\beta_{10}-\beta_{11}-\alpha_{12}-\alpha_{11}}{\alpha_{10}}, 1\right) .
\end{aligned}
$$


3.4. Stability of the Equilibrium Points. An evolutionary stable strategy (ESS) corresponds to an asymptotically stable fixed point. In this section, we analyze the stability of the equilibrium points by the Jacobian matrix $J$ [19].
Denoting the right of equation (11) by $M(x, y, z)$, $S(x, y, z)$, and $U(x, y, z)$, respectively, then the Jacobian matrix of equation (11) is obtained as

$$
J(x, y, z)=\left(\begin{array}{lll}
\frac{\partial M(x, y, z)}{\partial x} & \frac{\partial M(x, y, z)}{\partial y} & \frac{\partial M(x, y, z)}{\partial z} \\
\frac{\partial S(x, y, z)}{\partial x} & \frac{\partial S(x, y, z)}{\partial y} & \frac{\partial S(x, y, z)}{\partial z} \\
\frac{\partial U(x, y, z)}{\partial x} & \frac{\partial U(p, x, y)}{\partial y} & \frac{\partial U(x, y, z)}{\partial z}
\end{array}\right)=\left[\begin{array}{lll}
j_{11} & j_{12} & j_{13} \\
j_{21} & j_{22} & j_{23} \\
j_{31} & j_{32} & j_{33}
\end{array}\right],
$$

where $j_{11}=(1-2 x)\left(y \alpha_{10}+z \alpha_{11}+\alpha_{12}-\beta_{10}+\beta_{11}-\beta_{12}\right)$, $j_{12}=x(1-x) \alpha_{10} j_{13}=x(1-x) \alpha_{11}, j_{21}=y(1-y) \alpha_{21} j_{22}=$ $(1-2 y)\left[\alpha_{20}+x \alpha_{21}+z \alpha_{22}-\beta_{20}-\beta_{21}-\beta_{22}-\beta_{23}\right], \quad j_{23}=$ $y(1-y) \alpha_{22}, j_{31}=z(1-z) \alpha_{31}, j_{32}=z(1-z) \alpha_{32}$, and $j_{33}=$ $(1-2 z)\left[\alpha_{30}+x \alpha_{31}+y \alpha_{32}+\beta_{31}-\beta_{30}\right]$.

Next, we determine the local stability of the dynamic system (11) by analyzing the sign of the determinant of matrix $J$ and the trace at the nine equilibrium points. If the determinant of matrix $J \operatorname{det} J$ is positive and the trace $\operatorname{tr} J$ is negative, then the evolutionary equilibrium is a local stable point, which can be interpreted as the ESS [19]. The judgment criteria is written as

$$
\left\{\begin{array}{l}
\operatorname{det} J=\left|\begin{array}{lll}
j_{11} & j_{12} & j_{13} \\
j_{21} & j_{22} & j_{23} \\
j_{31} & j_{32} & j_{33}
\end{array}\right|>0, \\
\operatorname{tr} J=j_{11}+j_{22}+j_{33}<0 .
\end{array}\right.
$$

(1) $E_{1}(0,0,0)$

$$
\left\{\begin{array}{l}
\operatorname{det} J=\left(\alpha_{12}-\beta_{10}+\beta_{11}-\beta_{12}\right)\left(\alpha_{20}-\beta_{20}-\beta_{21}-\beta_{22}-\beta_{23}\right)\left(\alpha_{30}+\beta_{31}-\beta_{30}\right), \\
\operatorname{tr} J=\alpha_{12}+\alpha_{20}+\alpha_{30}+\beta_{31}-\beta_{10}+\beta_{11}-\beta_{12}-\beta_{20}-\beta_{21}-\beta_{22}-\beta_{23}-\beta_{30} .
\end{array}\right.
$$

(2) $E_{2}(0,0,1)$

$$
\left\{\begin{array}{l}
\operatorname{det} J=-\left(\alpha_{11}+\alpha_{12}-\beta_{10}+\beta_{11}-\beta_{12}\right)\left(\alpha_{20}+\alpha_{22}-\beta_{20}-\beta_{21}-\beta_{22}-\beta_{23}\right)\left(\alpha_{30}+\beta_{31}-\beta_{30}\right), \\
\operatorname{tr} J=\alpha_{11}+\alpha_{12}+\alpha_{20}+\alpha_{22}-\alpha_{30}-\beta_{10}+\beta_{11}-\beta_{12}-\beta_{20}-\beta_{21}-\beta_{22}-\beta_{23}+\beta_{30}-\beta_{31} .
\end{array}\right.
$$

(3) $E_{3}(0,1,0)$

$$
\left\{\begin{array}{l}
\operatorname{det} J=-\left(\alpha_{10}+\alpha_{12}-\beta_{10}+\beta_{11}-\beta_{12}\right)\left(\alpha_{20}-\beta_{20}-\beta_{21}-\beta_{22}-\beta_{23}\right)\left(\alpha_{30}+\alpha_{32}+\beta_{31}-\beta_{30}\right), \\
\operatorname{tr} J=\alpha_{10}+\alpha_{12}-\beta_{10}+\beta_{11}-\beta_{12}-\alpha_{20}+\beta_{20}+\beta_{21}+\beta_{22}+\beta_{23}+\alpha_{30}+\alpha_{32}+\beta_{31}-\beta_{30} .
\end{array}\right.
$$


(4) $E_{4}(0,1,1)$

$$
\left\{\begin{array}{l}
\operatorname{det} J=\left(\alpha_{10}+\alpha_{11}+\alpha_{12}-\beta_{10}+\beta_{11}-\beta_{12}\right)\left(\alpha_{20}+\alpha_{22}-\beta_{20}-\beta_{21}-\beta_{22}-\beta_{23}\right)\left(\alpha_{30}+\alpha_{32}+\beta_{31}-\beta_{30}\right), \\
\operatorname{tr} J=\alpha_{10}+\alpha_{11}+\alpha_{12}-\beta_{10}+\beta_{11}-\beta_{12}-\alpha_{20}-\alpha_{22}+\beta_{20}+\beta_{21}+\beta_{22}+\beta_{23}-\alpha_{30}-\alpha_{32}-\beta_{31}+\beta_{30} .
\end{array}\right.
$$

(5) $E_{5}(1,0,0)$

$$
\left\{\begin{array}{l}
\operatorname{det} J=-\left(\alpha_{12}-\beta_{10}+\beta_{11}-\beta_{12}\right)\left(\alpha_{20}+\alpha_{21}-\beta_{20}-\beta_{21}-\beta_{22}-\beta_{23}\right)\left(\alpha_{30}+\alpha_{31}+\beta_{31}-\beta_{30}\right), \\
\operatorname{tr} J=-\alpha_{12}+\beta_{10}-\beta_{11}+\beta_{12}+\alpha_{20}+\alpha_{21}-\beta_{20}-\beta_{21}-\beta_{22}-\beta_{23}+\alpha_{30}+\alpha_{31}+\beta_{31}-\beta_{30} .
\end{array}\right.
$$

(6) $E_{6}(1,0,1)$

$$
\left\{\begin{array}{l}
\operatorname{det} J=\left(\alpha_{11}+\alpha_{12}-\beta_{10}+\beta_{11}-\beta_{12}\right)\left(\alpha_{20}+\alpha_{21}+\alpha_{22}-\beta_{20}-\beta_{21}-\beta_{22}-\beta_{23}\right)\left(\alpha_{30}+\alpha_{31}+\beta_{31}-\beta_{30}\right), \\
\operatorname{tr} J=-\alpha_{11}-\alpha_{12}+\beta_{10}-\beta_{11}+\beta_{12}+\alpha_{20}+\alpha_{21}+\alpha_{22}-\beta_{20}-\beta_{21}-\beta_{22}-\beta_{23}-\alpha_{30}-\alpha_{31}-\beta_{31}+\beta_{30} .
\end{array}\right.
$$

(7) $E_{7}(1,1,0)$

$$
\left\{\begin{array}{l}
\operatorname{det} J=\left(\alpha_{10}+\alpha_{12}-\beta_{10}+\beta_{11}-\beta_{12}\right)\left(\alpha_{20}+\alpha_{21}-\beta_{20}-\beta_{21}-\beta_{22}-\beta_{23}\right)\left(\alpha_{30}+\alpha_{31}+\alpha_{32}+\beta_{31}-\beta_{30}\right), \\
\operatorname{tr} J=-\alpha_{11}-\alpha_{12}+\beta_{10}-\beta_{11}+\beta_{12}-\alpha_{20}-\alpha_{21}+\beta_{20}+\beta_{21}+\beta_{22}+\beta_{23}+\alpha_{30}+\alpha_{31}+\alpha_{32}+\beta_{31}-\beta_{30} .
\end{array}\right.
$$

(8) $E_{8}(1,1,1)$

$$
\left\{\begin{array}{l}
\operatorname{det} J=-\left(\alpha_{10}+\alpha_{11}+\alpha_{12}-\beta_{10}+\beta_{11}-\beta_{12}\right)\left(\alpha_{20}+\alpha_{21}+\alpha_{22}-\beta_{20}-\beta_{21}-\beta_{22}-\beta_{23}\right)\left(\alpha_{30}+\alpha_{31}+\alpha_{32}+\beta_{31}-\beta_{30}\right), \\
\operatorname{tr} J=-\alpha_{11}-\alpha_{12}+\beta_{10}-\beta_{11}+\beta_{12}-\alpha_{20}-\alpha_{21}+\beta_{20}+\beta_{21}+\beta_{22}+\beta_{23}-\alpha_{30}-\alpha_{31}-\alpha_{32}-\beta_{31}+\beta_{30} .
\end{array}\right.
$$

Next, we resort to the numerical experiment to illustrate the equilibrium points of the tripartite evolutionary game and carry out a sensitivity analysis.

\section{Numerical Experiment}

In this section, numerical experiment is conducted to stimulate and analyze the ESS of the tripartite game for Internet public opinion events.

4.1. Parameter Setting. The parameters of the evolutionary game model are given in Table 3 to facilitate our numerical studies, where $\alpha$ and $\beta$ denote revenues and costs, respectively.

4.2. Analysis of the Equilibrium Points. Based on the parameter values in Table 3, 15 equilibrium points are obtained by solving equation (11) as follows: $(0,0,0),(0,0,1),(0,1,0)$, $(1,0,0),(0,1,1),(1,1,0),(1,0,1),(1,1,1),(0,-1 / 8,-3.4)$, $(1,-3 / 8,-4.4),(-1 / 2,0,-16 / 2),(-21 / 2,1,-19 / 2),(-17 / 5$, $-16 / 3,0), \quad(-22 / 5,-18 / 3,1), \quad$ and $\quad(49.3 / 51,11.2 / 51$, $-265.2 / 51)$.

Since mixed strategy equilibrium points should satisfy $x^{*} \in(0,1), y^{*} \in(0,1)$, and $z^{*} \in(0,1)$, the seven points $(0,-1 / 8,-3.4), \quad(1,-3 / 8,-4.4), \quad(-1 / 2,0,--16 / 2)$, $(-21 / 2,1,-19 / 2), \quad(-17 / 5,-16 / 3,0), \quad(-22 / 5,-18 / 3,1)$, and $(49.3 / 51,11.2 / 2,-265.2 / 51)$ are not meaningful mixed strategy equilibrium points given the negative values therein. As such, among the aforesaid 15 equilibrium points, only 8 meaningful equilibrium points remain, $(0,0,0),(0,0,1)$, $(0,1,0),(1,0,0),(0,1,1),(1,1,0),(1,0,1)$, and $(1,1,1)$, which are the pure strategy equilibrium points.

Next, we assess the stability of these eight equilibrium points. According to equation (17), their Jacobian matrices are calculated as shown below: 
TABLE 3: The parameters of the evolutionary game model.

\begin{tabular}{|c|c|c|}
\hline Participants & & Parameters \\
\hline Internet medium & & $\alpha_{10}=2, \alpha_{11}=8, \alpha_{12}=4, \beta_{10}=13, \beta_{11}=1, \beta_{12}=6$ \\
\hline $\begin{array}{l}\text { University students } \\
\text { University administration }\end{array}$ & Profit and loss value (unit: ten thousand yuan) & $\begin{array}{c}\alpha_{20}=1, \alpha_{21}=0.8, \alpha_{22}=0.3, \beta_{20}=0.5, \beta_{21}=1, \beta_{22}=12, \\
\beta_{23}=2 \\
\alpha_{30}=5, \alpha_{31}=6\end{array}$ \\
\hline
\end{tabular}

$$
\begin{aligned}
& \left\{\begin{array}{l}
\operatorname{det} J(0,0,0)=\left|\begin{array}{ccc}
16 & 0 & 0 \\
0 & 3.4 & 0 \\
0 & 0 & 13
\end{array}\right|=707.2>0, \\
\operatorname{trJ}(0,0,0)=32.4>0,
\end{array}\right. \\
& \left\{\begin{array}{l}
\operatorname{det} J(0,0,1)=\left|\begin{array}{ccc}
18 & 0 & 0 \\
0 & 4.4 & 0 \\
0 & 0 & -13
\end{array}\right|=-1029.6<0, \\
\operatorname{trJ}(0,0,1)=9.4>0,
\end{array}\right. \\
& \left\{\begin{array}{l}
\operatorname{det} J(0,1,0)=\left|\begin{array}{ccc}
19 & 0 & 0 \\
0 & -3.4 & 0 \\
0 & 0 & 21
\end{array}\right|=-1356.6<0, \\
\operatorname{trJ}(0,1,0)=36.6>0,
\end{array}\right. \\
& \left\{\begin{array}{l}
\operatorname{det} J(1,0,0)=\left|\begin{array}{ccc}
-16 & 0 & 0 \\
0 & 4.4 & 0 \\
0 & 0 & 15
\end{array}\right|=-1056<0, \\
\operatorname{tr} J(1,0,0)=3.4>0,
\end{array}\right. \\
& \left\{\begin{array}{l}
\operatorname{det} J(0,1,1)=\left|\begin{array}{ccc}
21 & 0 & 0 \\
0 & -4.4 & 0 \\
0 & 0 & -21
\end{array}\right|=1950.4>0, \\
\operatorname{trJ}(0,1,1)=-4.4<0,
\end{array}\right. \\
& \left\{\begin{array}{l}
\operatorname{det} J(1,1,0)=\left|\begin{array}{ccc}
-19 & 0 & 0 \\
0 & -4.4 & 0 \\
0 & 0 & 23
\end{array}\right|=1922.8>0, \\
\operatorname{trJ}(1,1,0)=-0.4<0,
\end{array}\right. \\
& \left\{\begin{array}{l}
\operatorname{det} J(1,0,1)=\left|\begin{array}{ccc}
-18 & 0 & 0 \\
0 & 5.4 & 0 \\
0 & 0 & -15
\end{array}\right|=1458>0, \\
\operatorname{tr} J(1,0,1)=-27.6<0,
\end{array}\right. \\
& \left\{\begin{array}{l}
\operatorname{det} J(1,1,1)=\left|\begin{array}{ccc}
-21 & 0 & 0 \\
0 & -5.4 & 0 \\
0 & 0 & -23
\end{array}\right|=-2608.2<0, \\
\operatorname{trJ}(1,1,1)=-49.4<0 .
\end{array}\right.
\end{aligned}
$$

Given the criteria given in equation (17), we have the evolutionary equilibrium stability analysis result in Table 4.
TABLE 4: Evolutionary equilibrium stability analysis.

\begin{tabular}{lccc}
\hline Evolutionary equilibrium & detJ & trJ & Local stability \\
\hline$(0,0,0)$ & + & + & Unsteady \\
$(0,0,1)$ & - & + & Saddle point \\
$(0,1,0)$ & - & + & Saddle point \\
$(1,0,0)$ & - & + & Saddle point \\
$(0,1,1)$ & + & - & ESS \\
$(1,1,0)$ & + & - & ESS \\
$(1,0,1)$ & + & - & ESS \\
$(1,1,1)$ & - & - & Saddle point \\
\hline
\end{tabular}

From Table 4, we can know that $(0,1,1),(1,1,0)$, and $(1,0,1)$ are currently ESS.

4.3. Sensitivity Analysis. In this section, sensitivity analyses are conducted to show the effect of the three participants' behavior on the evolutionary path and evolutionary equilibrium strategies. Next, we successively examine the impact of the initial probability distributions as well as the three players' revenue and cost parameters.

4.3.1. Effects of the Initial Probability Distributions. In sensitivity analysis, we first analyze the effects of the initial probability distributions. Five groups of the initial probability distributions are set as shown in Table 5 . The revenue and cost parameters are set as per Table 3 in Section 4.1. The results are visually displayed in Figures 1-3.

Figure 1 shows that as the initial probability of the Internet medium taking the "reporting $m_{1}$ " strategy increases, the system will increase its rate of evolution toward $\left(m_{1}, s_{1}\right.$, $u_{1}$ ). This result indicates that a higher probability of the network medium reporting the event is conducive to more effective guidance of the university administration in the evolution of the public opinion event.

Figure 2 shows that the evolution rate of the system towards $\left(m_{1}, s_{1}, u_{1}\right)$ will be enhanced with a higher initial probability of university students' participating in the Internet public opinion event. This implies that a higher probability of university students" "participation" in spreading the words is beneficial for the university administration to effectively guide the development of the online public opinion event.

According to Figure 3, a higher initial probability of the university administration's choice of the "positive response" strategy allows the system to evolve more quickly to situation $\left(m_{1}, s_{1}, u_{1}\right)$. It indicates that the university administration should actively respond to the Internet public opinion event in order to effectively guide its evolution. 
TABLE 5: Five groups of the initial probability distributions.

\begin{tabular}{llllll}
\hline Participants & \multicolumn{5}{c}{ Probability } \\
\hline Internet medium & $x=0.1$ & $x=0.3$ & $x=0.5$ & $x=0.7$ & $x=0.9$ \\
University students & $y=0.1$ & $y=0.3$ & $y=0.5$ & $y=0.7$ & $y=0.9$ \\
University & $z=0.1$ & $z=0.3$ & $z=0.5$ & $z=0.7$ & $z=0.9$ \\
administration & &
\end{tabular}

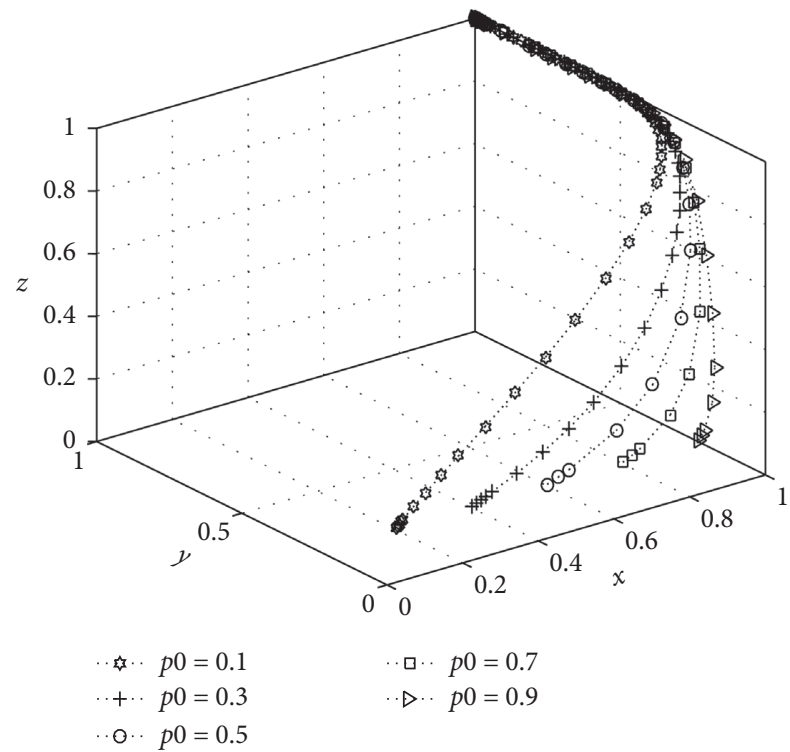

FIGURE 1: Effects of the initial distributions (Internet medium).

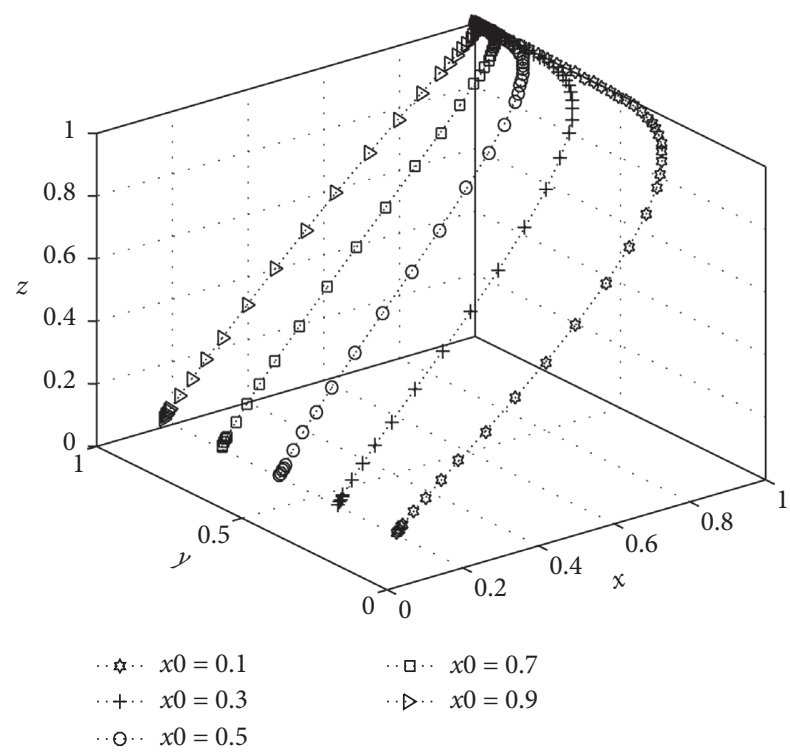

FIGURE 2: Effects of the initial distribution (university students).

4.3.2. Effects of the Internet Medium Parameters. This section discusses the effects of the benefits and costs of the Internet medium. The corresponding benefit and cost parameters are listed in Table 6 where $\alpha$ and $\beta$ denote, respectively, the revenues and costs. The results are illustrated in Figures 4-7.

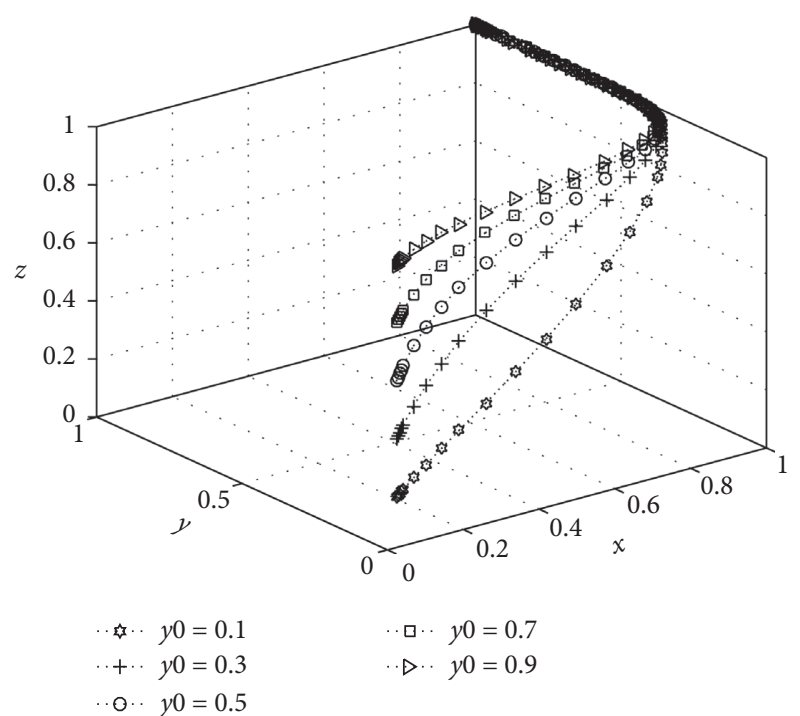

Figure 3: Effects of the initial distribution (university administration).

TABle 6: Parameter set of the network media.

\begin{tabular}{lccc}
\hline Parameters & \multicolumn{3}{c}{ Parameter values } \\
\hline$\alpha_{10}$ & $\alpha_{10}=1$ & $\alpha_{10}=3$ & $\alpha_{10}=5$ \\
$\alpha_{11}$ & $\alpha_{11}=1$ & $\alpha_{11}=2$ & $\alpha_{11}=3$ \\
$\alpha_{12}$ & $\alpha_{12}=2$ & $\alpha_{12}=8$ & $\alpha_{12}=10$ \\
$\beta_{10}$ & $\beta_{10}=4$ & $\beta_{10}=14$ & $\beta_{10}=25$ \\
$\beta_{11}$ & $\beta_{11}=3$ & $\beta_{11}=13$ & $\beta_{11}=23$ \\
$\beta_{12}$ & $\beta_{12}=1$ & $\beta_{12}=5$ & $\beta_{12}=10$ \\
\hline
\end{tabular}

According to Figure 4, we know that the evolution rate of the system to $\left(m_{1}, s_{1}, u_{1}\right)$ increases with a higher revenue of the network medium. It shows that a higher revenue for the Internet medium motivates it to adopt the reporting strategy to capitalize on the event to maximize its payoff.

Figures 5 and 7 demonstrate that higher costs of information handling, tracing, and social disorder slow down the evolution of the system to $\left(m_{1}, s_{1}, u_{1}\right)$. As a matter of fact, once these costs exceed certain thresholds, the Internet medium prefers to choose the "No-reporting $m_{2}$ " strategy instead.

On the other hand, Figure 6 demonstrates that an increasing cost of user churn will only slow down the evolution of the system to $\left(m_{1}, s_{1}, u_{1}\right)$ without diverting the Internet medium away from the "reporting $m_{1}$ " strategy.

4.3.3. Effects of the University Student Parameters. Next, we analyze the effects of university student revenue and cost parameters. The corresponding parameter values are listed in Table 7 , and the numerical study results are shown in Figures $8-12$, where $\alpha$ and $\beta$ denote, respectively, the university students' revenues and costs.

According to Figure 8, the system will evolve more quickly from the equilibrium point $(1,0,1)$ (i.e., $\left.\left(m_{1}, s_{2}, u_{1}\right)\right)$ to the asymptotically stable equilibrium point $(1,1,1)$ (i.e., $\left.\left(m_{1}, s_{1}, u_{1}\right)\right)$ when the revenues of university students are 




… $\alpha_{10}=1, \alpha_{11}=1, \alpha_{12}=2$

... $\alpha_{10}=3, \alpha_{11}=2, \alpha_{12}=8$

○. $\alpha_{10}=5, \alpha_{11}=3, \alpha_{12}=10$

FIGURE 4: The effect of the benefits.

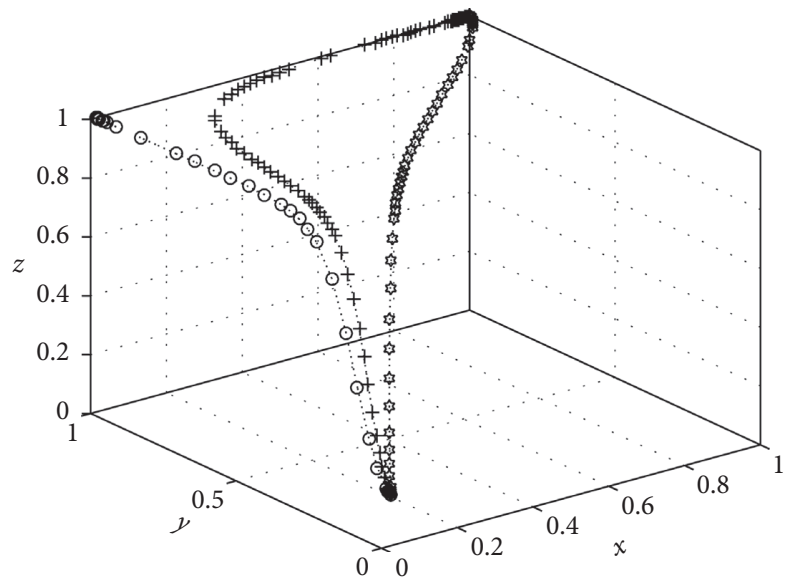

4. $\beta_{10}=4$

$+\cdot \beta_{10}=14$

○. $\beta_{10}=25$

Figure 5: The effect of $\beta_{10}$.

high enough and increase steadily. When the revenues from participation are sufficiently low, the students will take the "No-participation $s_{2}$ " strategy instead. In this case, the optimal situation of the whole game is $\left(m_{1}, s_{2}\right.$, $\left.u_{1}\right)$. But, it is not stable and causes reputation damage to the university. Accordingly, the university administration has motivation to adopt some measures so that it can influence the Internet public opinion. When the revenues of the students increase beyond certain thresholds, the optimal situation of the system changes from $\left(m_{1}, s_{2}, u_{1}\right)$ to $\left(m_{1}, s_{1}, u_{1}\right)$. Therefore, one can conclude that improving students' revenues is an effective measure to influence the dynamics of Internet public opinions.

From Figures 9-12, we can see that the system slowly evolves from $\left(m_{1}, s_{2}, u_{1}\right)$ to $\left(m_{1}, s_{1}, u_{1}\right)$ due to higher costs of searching time, individual misjudgment, university

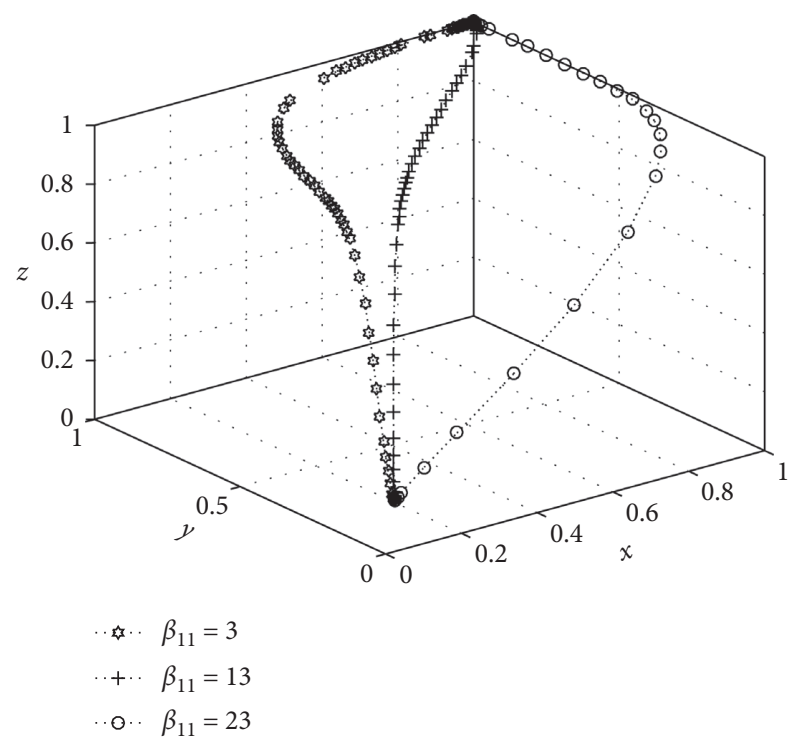

FIgURE 6: The effect of $\beta_{11}$.

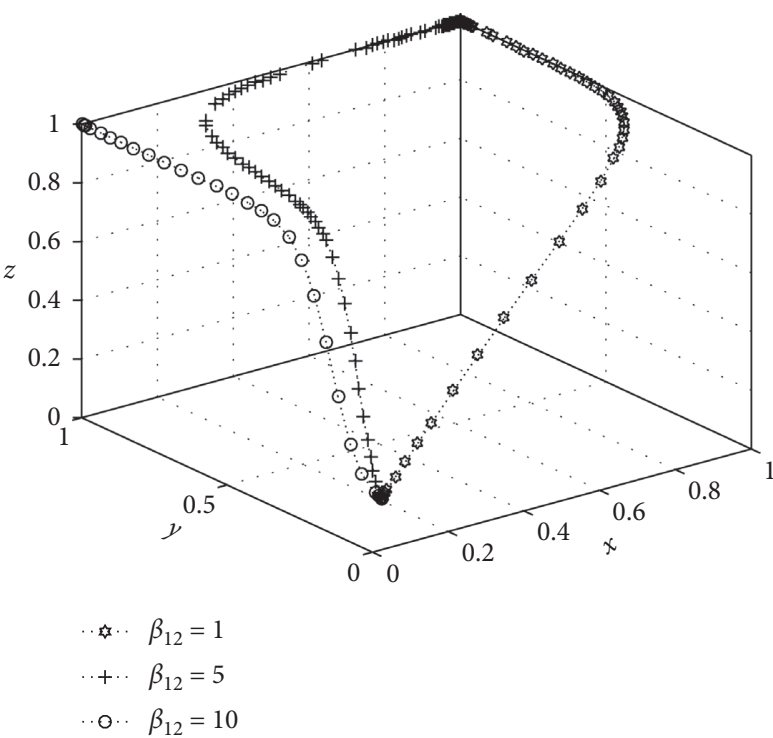

FIgURE 7: The effect of $\beta_{12}$.

disciplining, and social disorder. In Figures 9-11, they may even select not to participate in the event if the relevant costs are sufficiently high. This result implies that the higher the student costs, the greater the chance that students will choose the "No-particitation $s_{2}$ " strategy.

\subsubsection{Effect of the University Administration Parameters.} The effects of university revenue and cost parameters are investigated in this section. Table 8 lists the relevant parameter values, and the resulting figures are given in Figures 13-15, where $\alpha$ and $\beta$ denote, respectively, the university administration's revenues and costs.

According to Figures 13-15, it is crucial that the university administration balances between the cost from 
TABle 7: Parameter values of university students.

\begin{tabular}{lccc}
\hline Parameters & & Parameter values & \\
\hline$\alpha_{20}$ & $\alpha_{20}=4$ & $\alpha_{20}=6$ & $\alpha_{20}=8$ \\
$\alpha_{21}$ & $\alpha_{21}=0$ & $\alpha_{21}=1$ & $\alpha_{21}=2$ \\
$\alpha_{22}$ & $\alpha_{22}=0$ & $\alpha_{22}=1$ & $\alpha_{22}=2$ \\
$\beta_{20}$ & $\beta_{10}=0.8$ & $\beta_{10}=1$ & $\beta_{10}=1.5$ \\
$\beta_{21}$ & $\beta_{21}=0.3$ & $\beta_{21}=0.6$ & $\beta_{21}=1$ \\
$\beta_{22}$ & $\beta_{22}=0.5$ & $\beta_{22}=1$ & $\beta_{22}=2$ \\
$\beta_{23}$ & $\beta_{23}=1$ & $\beta_{23}=2$ & $\beta_{23}=3$ \\
\hline
\end{tabular}

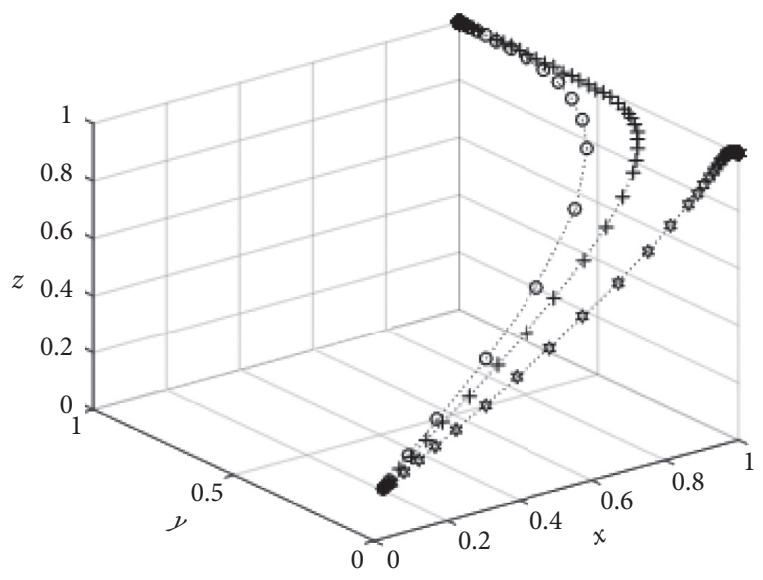

‥ $\alpha_{20}=4, \alpha_{21}=0, \alpha_{22}=0$

.. ‥ $\alpha_{20}=6, \alpha_{21}=1, \alpha_{22}=1$

.०. $\alpha_{20}=8, \alpha_{21}=2, \alpha_{22}=2$

Figure 8: The effect of the revenue.

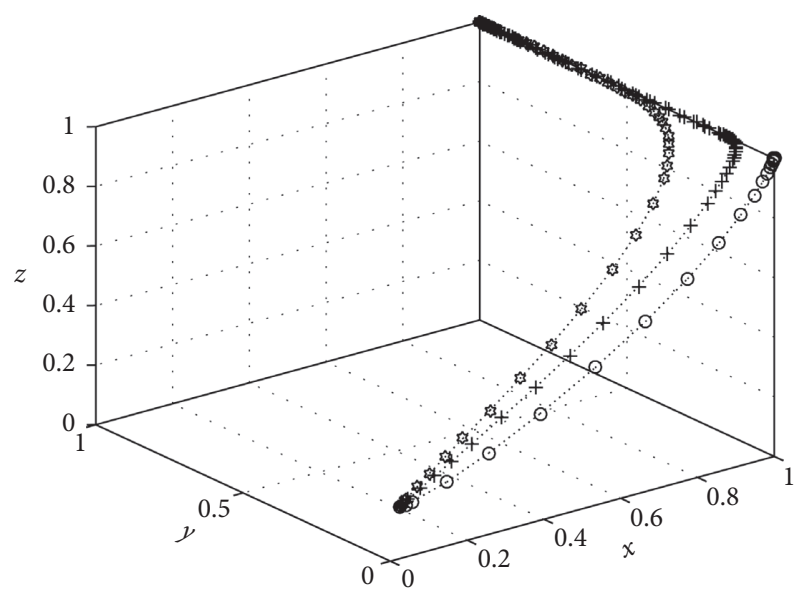

. $\quad \beta_{20}=0.8$

-.. $\beta_{20}=1$

.. ०. $\beta_{20}=1.5$

FIgUre 9: The effect of $\beta_{20}$.

damaged university reputation and the relevant handling costs (i.e., manpower, material resources and time in topics, and handling information in time). When the university

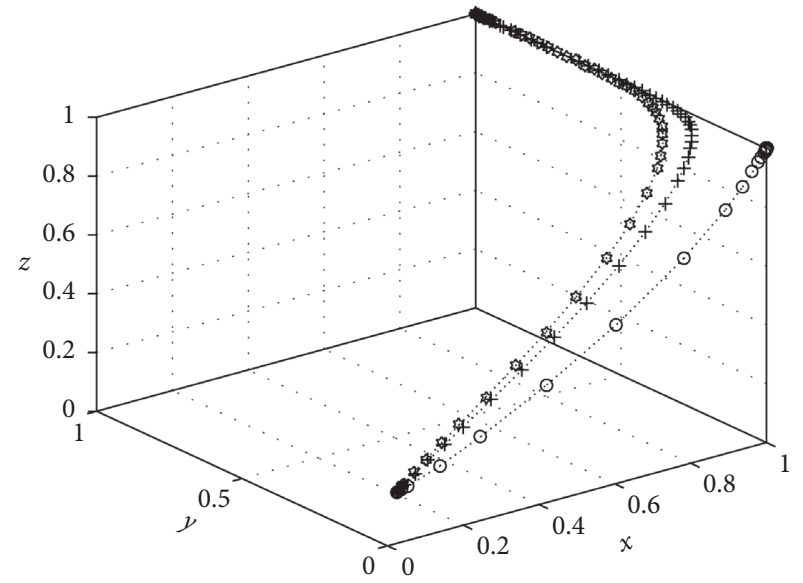

- $\beta_{21}=0.3$

$+\cdot \beta_{21}=0.6$

... $\beta_{21}=1$

Figure 10: The effect of $\beta_{21}$.

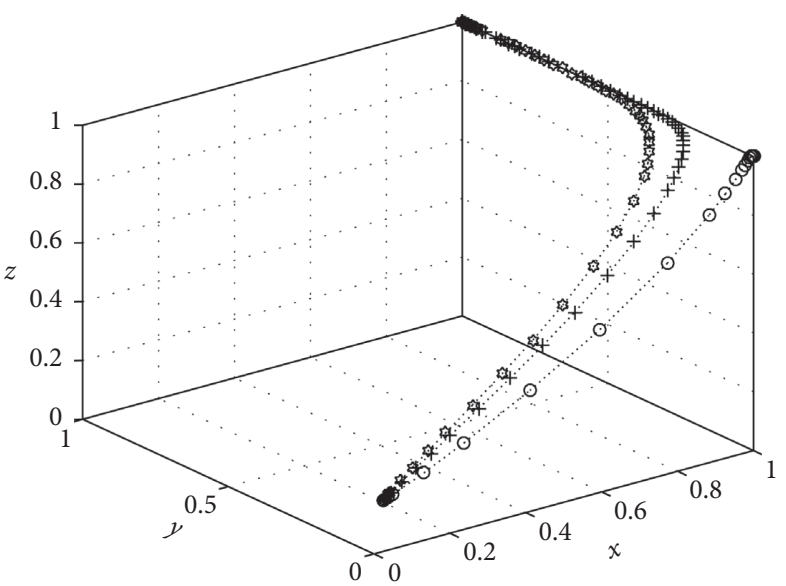

- $\beta_{22}=0.5$

$+\cdot \beta_{22}=1$

.०. $\beta_{22}=2$

Figure 11: The effect of $\beta_{22}$.

administration can efficiently guide the evolution of the public opinion, they prefer to adopt the "positive response $u_{1}$ " strategy to enhance university reputation. 


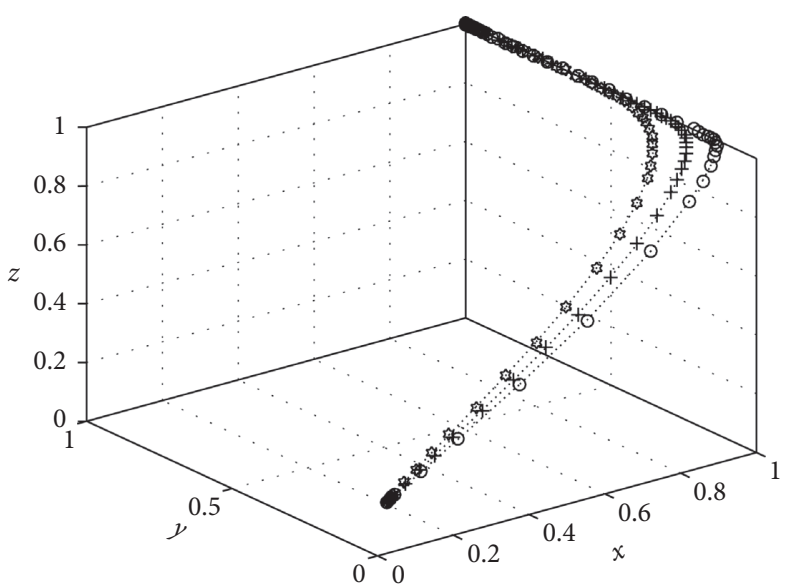

- . $\beta_{23}=1$

$+\cdot \beta_{23}=2$

○. $\beta_{23}=3$

Figure 12: The effect of $\beta_{23}$.

TABle 8: Parameter set of the university.

\begin{tabular}{lccc}
\hline Parameters & & Parameter values & \\
\hline$\alpha_{30}$ & $\alpha_{30}=12$ & $\alpha_{30}=14$ & $\alpha_{30}=16$ \\
$\alpha_{31}$ & $\alpha_{31}=2$ & $\alpha_{31}=4$ & $\alpha_{31}=6$ \\
$\alpha_{32}$ & $\alpha_{32}=8$ & $\alpha_{32}=10$ & $\alpha_{32}=12$ \\
$\beta_{30}$ & $\beta_{30}=5$ & $\beta_{30}=7$ & $\beta_{30}=19$ \\
$\beta_{31}$ & $\beta_{31}=6$ & $\beta_{31}=8$ & $\beta_{31}=10$ \\
\hline
\end{tabular}

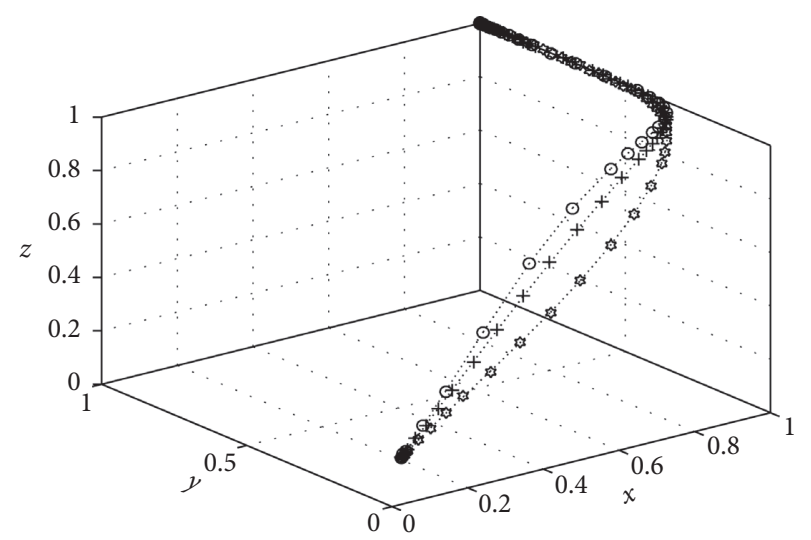

4. $\alpha_{30}=12, \alpha_{31}=2, \alpha_{32}=8$

$+\cdot \alpha_{30}=14, \alpha_{31}=4, \alpha_{32}=10$

○. . $\alpha_{30}=16, \alpha_{31}=6, \alpha_{32}=12$

Figure 13: The effect of the revenue.

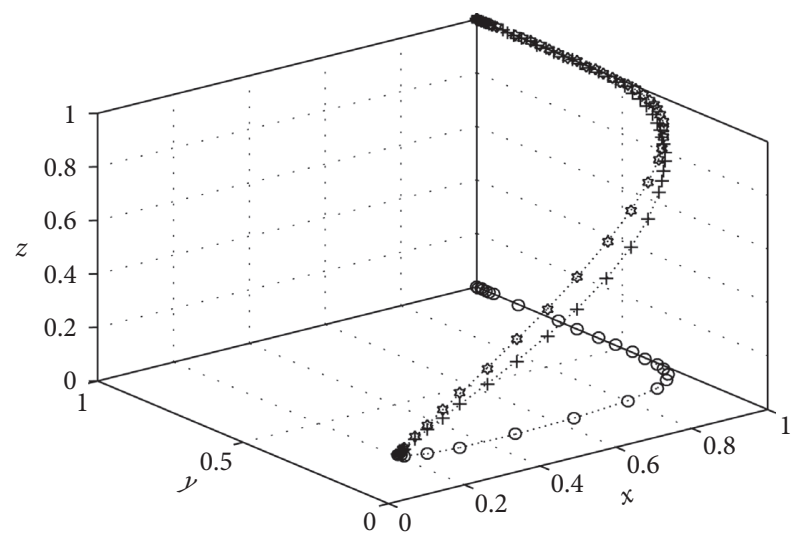

- $\quad \beta_{30}=5$

$+\cdot \beta_{30}=7$

○. $\beta_{30}=19$

Figure 14: The effect of $\beta_{30}$. 


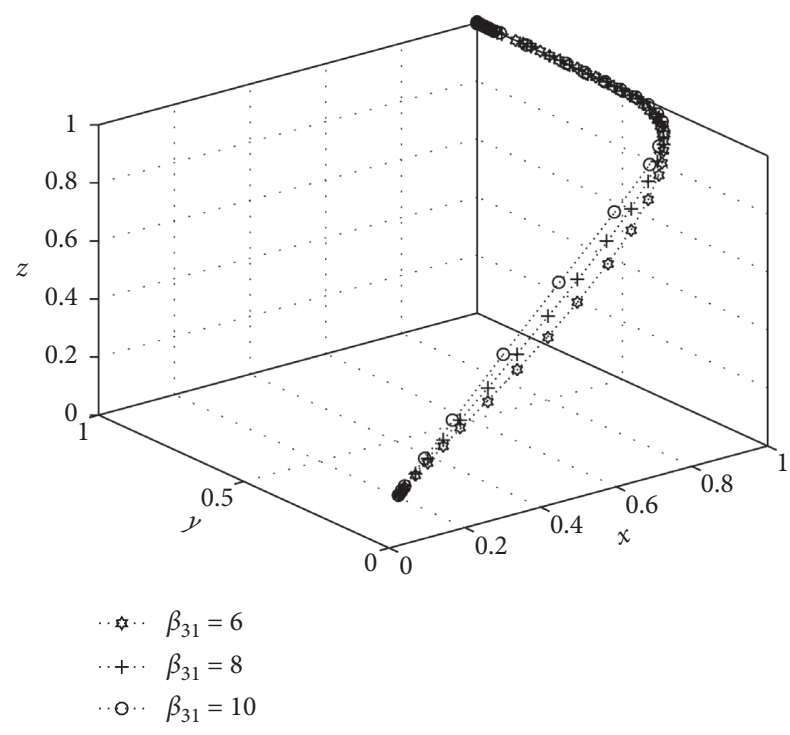

FIGURE 15: The effect of $\beta_{31}$.

\section{Conclusion}

This paper develops a tripartite evolutionary game model consisting of an Internet medium, university students as a group, and university administration to examine the process of Internet public opinion evolution within a university setting in China. The model is solved to obtain the differential dynamic equations and the equilibrium points. We then carry out a stability analysis of the equilibrium points. Through the analytic and numerical studies, we obtain the following results:

(1) The Internet medium, university students, and university administration continuously adjust their own strategies, and the dynamic evolution of the system finally reaches a stable situation at $(1,1,1)$ corresponding to each of the three players selecting their first strategy in the ESS situation $\left(m_{1}, s_{1}, u_{1}\right)$. More specially, if the three participants have higher probabilities of selecting their first strategy at the initial stage of the game, the evolutionary equilibrium $\left(m_{1}, s_{1}, u_{1}\right)$ arises more quickly.

(2) From the perspective of the Internet medium, they should adopt an objective and fair attitude toward reporting university Internet public opinion events based on socially responsible and industrial norms. The government, as the authority for public opinion supervision, should formulate appropriate laws and regulations to enhance its monitoring of online media communications. On the one hand, appropriate penalties should be given to irresponsible behavior of the network media to block reports. On the other hand, it is necessary to increase the revenues of truthful reporting and other Internet media operations, as well as reduce the cost of publishing and following up relevant information by the medium.

(3) University students, as the main recipients and spreaders of university Internet public opinions, should be educated and guided by the university administration. University students should improve their online media literacy to differentiate rational arguments from miscellaneous and false Internet information and be educated about the negative impact of disseminating disinformation about sensitive events, thereby reducing their participation benefits. In addition, online real-name authentication improves the participation costs for university students and reduces spreading of rumors and disinformation about public opinion events.

(4) Diverse information about an Internet public opinion event can rapidly spread through various social media channels (i.e., Google, Baidu, Weibo, Blog, and WeChat). Facing an Internet public opinion event, the university administration should release truthful and comprehensive information in a timely manner. This is the best handling approach as it helps to maintain transparency in communicating facts to the public and prevent disinformation and rumors from spreading. In addition, timely release of truthful information can help to reduce costs of manpower, resources, and time in response to the event. Furthermore, the university administration can exploit big data analysis to better understand the dynamics of the public opinion evolution, thereby avoiding deterioration of the situation caused by undesirable spreading of disinformation about the event and minimizing subsequent negative impacts.

This research has its limitations and can be further extended along several directions. Firstly, this paper assumes that the players' payoff functions are independent of time. The implication of this assumption is that each participant's action at time $t+1$ only depends on the actions of all participants at time $t$. To better characterize the dynamics of public opinion events, it would be interesting to consider the case that the payoffs are functions of time $t$. Secondly, the proposed model consists of three participants, an Internet medium, university students as a group (a single player), and one university administration. In reality, there are often multiple players and multiple strategies in different types of participants. In this case, a networked evolutionary game model would be a more appropriate tool. To address this more complicated network structure, the novel methodologies and models furnished by Zhao et al. $[20,21]$ can be useful. We may establish a networked evolutionary game model for university public opinion events and employ the trajectory control [20] and strategy consensus method [21] to investigate different participants' strategies and derive useful managerial insights for different stakeholders in these events.

\section{Data Availability}

The data used to support the findings of this study are available from the corresponding author upon request.

\section{Conflicts of Interest}

The authors declare that they have no conflicts of interest. 


\section{Acknowledgments}

This work was supported in part by the Social Science Planning Foundation of Fujian Province under Grant FJ2018B132, in part by the Ministry of Education $\mathrm{Hu}-$ manities and Social Sciences Research Project under Grant 18JDSZ3035, in part by the Fujian Provincial Administrative Management Project under Grant 2018B01, and in part by Social Science Fund of Fujian Province, China under Grant FJ2019B031.

\section{References}

[1] People.com.cn. South China University of Technology tampered postgraduate entrance exam scores. https://baike.baidu. com/reference/23292912/8be7-ZbqivlwYj0_vOO8ojIC3Fwg VQDLCwmSBgjIdCXiYJ_JqIJtoyLBHUdl9ASjr9-CSgTvHJY_ ix7x1Bl4CuiZmeJDXxbw2aLOcjg6mv4nL9eY0LBx. 2020.

[2] Chinadaily.com. Foreign Students' Study Partners at the Shandong University. http://cnews.chinadaily.com.cn/a/ 201907/12/WS5d27fc99a310a6dd41e85925.html. 2020.

[3] B. Laxmidhar and S. Frank, "On spatial consensus formation is the Sznajd model different from a voter model?" International Journal of Modern Physics C, vol. 14, no. 19, pp. 1331-1354, 2003.

[4] G. Weisbuch, "Bounded confidence and social networks," The European Physical Journal B-Condensed Matter, vol. 38, no. 2, pp. 339-343, 2004.

[5] S. Fortunato, "Damage spreading and opinion dynamics on scale-free networks," Physica A: Statistical Mechanics and Its Applications, vol. 348, pp. 683-690, 2005.

[6] A. D. Mare and V. Latora, "Opinion formation models based on game theory," International Journal of Modern Physics C, vol. 18, no. 9, pp. 1377-1395, 2007.

[7] L. Cao and X. Li, "Mixed evolutionary strategies imply coexisting opinions on networks," Physical Review E, vol. 77, no. 1, p. 016108, 2008.

[8] D. Liu, W. Wang, and H. Li, "Evolutionary mechanism and information supervision of public opinions in internet emergency," Procedia Computer Science, vol. 17, pp. 973-980, 2013.

[9] D. Li, J. Ma, Z. Tian, and H. Zhu, "An evolutionary game for the diffusion of rumor in complex networks," Physica A: Statistical Mechanics and Its Applications, vol. 433, no. 433, pp. 51-58, 2015.

[10] H. Jiang, M. Qiang, and P. Lin, "Assessment of online public opinions on large infrastructure projects: a case study of the Three gorges project in China," Environmental Impact Assessment Review, vol. 61, pp. 38-51, 2016.

[11] C. Ai, B. Chen, L. He, K. Lai, and X. Qiu, "The national geographic characteristics of online public opinion propagation in China based on WeChat network," Geoinformatica, vol. 22, no. 2, pp. 311-334, 2018.

[12] Y. Wang, H. Li, J. Zuo, and Z. Wang, "Evolution of online public opinions on social impact induced by NIMBY facility," Environmental Impact Assessment Review, vol. 78, Article ID 106290, 2019.

[13] M. Johari, S.-M. Hosseini-Motlagh, and M. Rasti-Barzoki, "An evolutionary game theoretic model for analyzing pricing strategy and socially concerned behavior of manufacturers," Transportation Research Part E: Logistics and Transportation Review, vol. 128, pp. 506-525, 2019.
[14] Q. Li, C. Song, B. Wu, Y. Xiao, and B. Wang, "Social hotspot propagation dynamics model based on heterogeneous mean field and evolutionary games," Physica A: Statistical Mechanics and Its Applications, vol. 508, pp. 324-341, 2018.

[15] Y. Xiao, C. Song, and Y. Liu, "Social hotspot propagation dynamics model based on multidimensional attributes and evolutionary games," Communications in Nonlinear Science and Numerical Simulation, vol. 67, pp. 13-25, 2019.

[16] X. Yin, H. Wang, P. Yin, and H. Zhu, "Agent-based opinion formation modeling in social network: a perspective of social psychology," Physica A: Statistical Mechanics and Its Applications, vol. 532, p. 121786, 2019.

[17] M. Askarizadeh, B. Tork Ladani, and M. H. Manshaei, "An evolutionary game model for analysis of rumor propagation and control in social networks," Physica A: Statistical Mechanics and Its Applications, vol. 523, pp. 21-39, 2019.

[18] C. Li, F. Zhang, C. Cao, Y. Liu, and T. Qu, "Organizational coordination in sustainable humanitarian supply chain: an evolutionary game approach," Journal of Cleaner Production, vol. 219, pp. 291-303, 2019.

[19] F. Daniel, "Evolutionary games in economics," Econometrica, vol. 59, no. 3, pp. 637-666, 1991.

[20] G. Zhao and S. Fu, "Matrix approach to trajectory control of higher-order $k$-valued logical control networks," IET Control Theory \& Applications, vol. 11, no. 13, pp. 2110-2115, 2017.

[21] G. Zhao, H. Li, W. Sun et al., "Modelling and strategy consensus for a class of networked evolutionary games," International Journal of Systems Science, vol. 40, no. 1, pp. 1-10, 2018. 